\title{
Editorials
}

\section{The effect of perioperative beta-blockers on patients undergoing noncardiac surgery - is the answer in?}

\author{
P.J. Devereaux MD, ${ }^{*}$ Kate Leslie MB BS MD M EPi, $†$ Homer Yang MD $\ddagger$
}

"Those who ignore the past are destined to repeat it"

$\mathrm{D}$ R. Rapchuk and colleagues in this edition of the journal report their study which evaluated the use of beta-blockers among, "suitable candidates for perioperative betablockade." The authors state, "perioperative betablockade reduces morbidity and mortality in noncardiac surgery." Other authors have also taken the position that the evidence is clear that perioperative beta-blockers are effective and have advocated their use in the perioperative setting. ${ }^{2-4}$ In this editorial we evaluate the conclusiveness of the evidence underlying the assertions of Dr. Rapchuk and others, and we use historical examples to provide insights.

\section{The perioperative beta-blocker evidence}

A recent meta-analysis provides the most comprehensive published overview of the short-term perioperative beta-blocker evidence in patients undergoing noncardiac surgery. ${ }^{5}$ This overview included 11 perioperative beta-blocker randomized controlled trials (RCTs) that fulfilled their eligibility criteria but only seven of these RCTs, which randomized a total of 656 patients, had patients who suffered a cardiovascular death or nonfatal myocardial infarction. The authors of the meta-analysis missed one nonfatal myocardial infarction in a RCT and incorrectly assumed the myocardial infarctions in another RCT were nonfatal. ${ }^{6}$
Considering these corrections there have been few patient important outcomes in these RCTs (i.e., 15 cardiac deaths and 18 nonfatal myocardial infarctions), and the majority of these events, 11 cardiac deaths and nine nonfatal myocardial infarctions, occurred in a single RCT by Poldermans and colleagues. $^{7}$ Despite the paucity of major events the meta-analysis suggests that perioperative beta-blockers reduce the relative risks of cardiac death and nonfatal myocardial infarction by $\geq 75 \%$.

A single RCT by Mangano and colleagues has evaluated the long-term effects of perioperative betablocker therapy in patients undergoing noncardiac surgery. ${ }^{8}$ This trial randomized 200 patients to atenolol or placebo for a maximum duration of seven postoperative days, and patients were then followed for a two-year period. The authors reported 30 deaths during follow-up and a $>50 \%$ reduction in the relative risk of death among patients who received perioperative atenolol. These results, however, did not include the six deaths that occurred during the period when patients were receiving the study drug. The possibility that beta-blockers may affect events after they have been discontinued is interesting but does not justify ignoring the events patients experience while taking the drug. When these events are appropriately included in the true intention-to-treat analysis the reduction in the risk of death with atenolol is no longer statistically significant.

From the Departments of Medicine and Clinical Epidemiology and Biostatics, ${ }^{*}$ McMaster University, Hamilton, Ontario, Canada; the Department of Anaesthesia and Pain Management, $†$ The Royal Melbourne Hospital, the Department of Pharmacology, $†$ University of Melbourne, Melbourne, Australia; and the Department of Anesthesia, $\ddagger$ University of Ottawa, Ottawa, Ontario, Canada.

Address correspondence to: Dr. P. J. Devereaux, Clinical Epidemiology and Biostatistics, McMaster University Health Sciences Centre, Room 2C8, 1200 Main Street West, Hamilton, Ontario L8N 3Z5, Canada. Phone: 905-525-9140, ext. 22804; Fax: 905-526-1353; E-mail: philipj@mcmaster.ca

Sources of funding: Dr. P. J. Devereaux is supported by a Canadian Institutes of Health Research, Senior Research Fellowship Award. Competing interests: Drs. P. J. Devereaux and Homer Yang are the Co Principal Investigators of the POISE trial. Dr. Kate Leslie is the POISE National Coordinator of Australia/Hong Kong/New Zealand/Singapore. 


\section{Interpretation of the perioperative beta-blocker evi- dence}

The perioperative beta-blocker evidence is encouraging, however, this evidence warrants cautious interpretation for a host of reasons. First, the perioperative beta-blocker evidence suggests large treatment effects (i.e., relative risk reductions $\geq 75 \%$ ). These results are inconsistent with beneficial cardiovascular therapies established in trials with 10,000 s of patients, which generally demonstrate moderate relative risk reductions in the order of 15 to $35 \% .{ }^{9}$ Cardiovascular therapies rarely demonstrate relative risk reductions greater than $35 \%$, because cardiovascular therapies usually only target a limited number of the multitude of pathogenic mechanisms underlying cardiovascular diseases, as is the case with perioperative beta-blockers. ${ }^{6}$

In the past, when initial small trials have claimed remarkably large benefits, subsequent trials typically demonstrated much more modest - or even no treatment effects. ${ }^{10,11}$ For example, a meta-analysis of RCTs of magnesium in acute myocardial infarction demonstrated a statistically significant $(P<0.001)>$ $50 \%$ reduction in death with approximately 1,500 patients randomized and a moderate number of deaths (i.e., 128 deaths, which is six times the total number of deaths in the perioperative beta-blocker RCTs). ${ }^{10}$ However, the subsequent RCT of approximately 60,000 patients showed no benefit; in fact there was a trend toward excess mortality with magnesium $(P=0.07) .{ }^{11}$

Second, there have been few major events in the perioperative beta-blocker RCTs. If perioperative betablockers are effective, given the arguments above, it is only plausible that they will demonstrate a moderate treatment effect (i.e., $15-35 \%$ relative risk reduction). Even assuming a high perioperative cardiovascular event rate of $10 \%$, trials will need at least 350 and ideally 650 events to convincingly demonstrate a $25 \%$ relative risk reduction. The perioperative beta-blocker RCT data include only 33 cardiovascular deaths and nonfatal myocardial infarctions, therefore constitute very limited evidence. Further, when the supportive evidence for an intervention comes from nothing more than a small number of trials with very few events, publication bias may seriously distort the evidence. ${ }^{12}$

Third, the results of the meta-analysis are completely dependent upon the results of a single RCT, by Poldermans and colleagues, that has several limitations. ${ }^{7}$ This RCT randomized only 112 patients and there were few events (i.e., 11 deaths and nine nonfatal myocardial infarctions). The RCT was unblinded, and research demonstrates that unblinded trials may introduce biases larger than the treatment effects that investigators typically seek to detect. ${ }^{13}$ The RCT was stopped because an interim analysis suggested large treatment effects (i.e., $100 \%$ relative risk reduction for nonfatal myocardial infarction and an $80 \%$ relative risk reduction for cardiac death). These treatment effects are implausibly large and they are inconsistent with the results of the beta-blocker RCTs in myocardial infarction and congestive heart failure that have randomized > 50,000 patients and have demonstrated moderate treatment effects (i.e., relative risk reductions of $15-35 \%){ }^{14,15}$ Studies that have documented patients suffering perioperative nonfatal myocardial infarctions despite perioperative beta-blocker therapy, refute the claim of a relative risk reduction of $100 \%$ for perioperative nonfatal myocardial infarctions with beta-blocker therapy. ${ }^{16}$ Finally, empiric evidence warns us to be skeptical of unexpectedly large treatment effects identified during interim analyses. ${ }^{17}$

Some authors have acknowledged limitations of the perioperative beta-blocker RCT evidence but have argued, "that given the strong efficacy in secondary prevention after myocardial infarction, additional perioperative studies are unnecessary."18 Our current knowledge of the pathophysiology of perioperative cardiovascular events is limited, and it is possible that the pathophysiology of perioperative myocardial infarctions is different from that of nonsurgical acute coronary syndromes. The rationale to treat patients with one condition based on knowledge that a drug is beneficial in another condition with similar underlying pathophysiology has, in an appreciable number of situations, proved disastrously wrong. For example, ST segment elevation myocardial infarction and non ST segment elevation myocardial infarction and unstable angina all share a very similar pathophysiology of an acute coronary thrombus. Thrombolytic therapy is beneficial in ST segment elevation myocardial infarction but not in the other conditions. ${ }^{19}$ Considering these issues and the potential complications (i.e., clinically significant bradycardia and hypotension) and cost of perioperative beta-blocker therapy it is not in the patients' best interest to assume that the secondary prevention postmyocardial infarction beta-blocker data tell us how to manage patients undergoing noncardiac surgery.

Dr. Rapchuk and colleagues evaluated the use of beta-blockers among, "suitable candidates for perioperative beta-blockade." "They state the "American College of Physicians (ACP) guidelines were used to determine which patients were candidates for perioperative beta-blockade." ${ }^{20}$ The ACP, however, does not have a guideline recommending perioperative betablocker therapy; rather the two authors of the guide- 
lines inserted their own recommendation for perioperative beta-blocker therapy after the guidelines were reviewed and approved by the ACP. The authors based their recommendation on the results of the RCT by Mangano and colleagues, which has a statistically non-significant result according to the true intention-to-treat analysis.

More recently the American College of Cardiology/American Heart Association (ACC/AHA) Task Force on practice guidelines for noncardiac surgery recommended beta-blocker therapy for patients with or at risk of coronary artery disease undergoing noncardiac surgery. ${ }^{21}$ The ACC/AHA Task Force did, however, state, "there are still very few randomized trials of medical interventions before noncardiac surgery to prevent perioperative cardiac complications, and they do not provide enough data from which to draw firm conclusions or recommendations." ${ }^{21}$ This highlights the need to distinguish a weak recommendation from a strong recommendation. The weak perioperative betablocker recommendations are similar to the prior weak recommendations to administer hormone replacement therapy to postmenopausal women. ${ }^{22}$ The results of the recent RCTs that have demonstrated that hormone replacement therapy increases the risk of coronary artery disease remind us why it is essential to undertake large definitive trials and avoid settling for weak recommendations. $^{23}$

\section{Need for a large definitive perioperative beta-blocker RCT}

As a result of the promising but uncertain perioperative beta-blocker evidence an international group of investigators have come together and set a goal to definitively establish the effect of perioperative betablocker therapy. This group has recently initiated a perioperative beta-blocker RCT that plans to randomize 10,000 patients. This trial is called the PeriOperative ISchemic Evaluation (POISE) trial and is funded by a Canadian Institutes of Health Research grant and a National Health and Medical Research Council grant from the Commonwealth Government of Australia. The POISE trial is randomizing patients to 30 days of perioperative metoprolol controlled released or placebo and the primary outcome is a composite of cardiovascular death, nonfatal myocardial infarction and nonfatal cardiac arrest. To date the trial has randomized over 2,000 patients in more than 100 centres in 11 countries.

\section{Conclusions}

The current RCT evidence evaluating the effects of perioperative beta-blocker therapy on short and long- term cardiovascular outcomes has significant limitations. The results of these RCTs should be viewed as promising but tempered with healthy skepticism. In keeping with this the perioperative beta-blocker recommendations are weak as the reliability of the underlying evidence is weak. The POISE trial will provide definitive evidence to determine the efficacy of perioperative beta-blocker therapy.

\section{Acknowledgements}

Dr. P. J. Devereaux contributed significantly to the concept and design of this paper. He wrote the first draft of the manuscript, and provided critical revisions to the manuscript. He also gave final approval of the submitted manuscript. Dr. Kate Leslie contributed significantly to the concept and design of this paper and provided critical revisions to the manuscript. She also gave final approval of the submitted manuscript. Dr. Homer Yang contributed significantly to the concept and design of this paper and provided critical revisions to the manuscript. He also gave final approval of the submitted manuscript.

\section{L'effet des bêta-blo- quants périopératoires sur les patients de chirurgie non cardiaque - avons-nous la réponse ?}

\section{«Ceux qui ignorent le passé sont appelés à le répéter»}

Dans le présent numéro du journal, le Dr Rapchuk et ses collègues présentent leur étude qui évalue l'usage des bêta-bloquants dans «des cas appropriés au bêtablocage périopératoire». ${ }^{1}$ Les auteurs affirment que «le bêta-blocage périopératoire réduit la morbidité et la mortalité en chirurgie non cardiaque». ${ }^{1}$ D'autres auteurs ont aussi conclu à l'efficacité clairement évidente des bêta-bloquants périopératoires et en ont conseillé l'usage dans le contexte périopératoire. ${ }^{2-4}$ Dans le présent commentaire éditorial, nous évaluons le caractère concluant de la preuve à l'origine des 
assertions du Dr Rapchuk et d'autres auteurs et présentons une critique des données à partir d'exemples historiques.

\section{La preuve de l'effet des bêta-bloquants périopéra- toires}

Une méta-analyse récente présente la revue la plus complète de l'usage périopératoire de courte durée des bêta-bloquants chez des patients de chirurgie non cardiaque. ${ }^{5}$ Elle comprend 11 études randomisées et contrôlées (ERC) de bêta-bloquants périopératoires répondant aux critères d'admissibilité, mais sept études seulement, auprès de 656 patients, comptent des décès à la suite de problèmes cardiovasculaires ou des infarctus myocardiques non fatals. Les auteurs de la méta-analyse ont manqué un infarctus myocardique non fatal dans l'une des ERC et ont présumé à tort que les infarctus myocardiques d'une autre ERC avaient été non fatals. ${ }^{6}$ Ces corrections faites, il y a eu peu de conséquences importantes pour les patients de ces ERC, c'est-à-dire 15 décès cardiaques et 18 infarctus myocardiques non fatals, et la majorité de ces incidents, 11 morts cardiaques et neuf infarctus myocardiques non fatals, sont survenus au cours d'une seule ERC réalisée par Poldermans et coll. ${ }^{7}$ Malgré le manque d'incidents majeurs, la méta-analyse conclut que les bêta-bloquants périopératoires réduisent les risques relatifs de mort cardiaque et d'infarctus myocardique non fatal de $\geq 75 \%$.

Une seule ERC, de Mangano et coll., a évalué les effets à long terme des bêta-bloquants périopératoires chez des patients de chirurgie non cardiaque. ${ }^{8}$ L'étude randomisait 200 patients pour de l'aténolol ou un placebo pendant une durée maximale de sept jours postopératoires. Les patients étaient suivis ensuite pendant deux ans. Les auteurs ont consigné 30 décès pendant le suivi et une réduction de $>50 \%$ du risque relatif de décès chez les patients sous aténolol périopératoire. Toutefois, ces résultats n'incluent pas les six décès survenus pendant que les patients recevaient le médicament expérimental. La possibilité que les bêta-bloquants aient un effet même après qu'on a arrêté de les prendre est intéressante, mais ne justifie pas l'exclusion des incidents qui ont touché les patients pendant qu'ils recevaient la médication. Quand on inclut ces incidents, comme il convient de le faire, à l'analyse d'intention thérapeutique, la réduction du risque de décès avec l'aténolol n'est plus statistiquement significative.

\section{L'interprétation de la preuve des bêta-bloquants péri- opératoires}

La preuve de l'effet des bêta-bloquants périopératoires est encourageante, bien qu'elle commande une interprétation prudente pour une foule de raisons. Premièrement, elle laisse croire à des effets thérapeutiques importants, comme une réduction du risque relatif de $\geq 75 \%$ ). Ces résultats contredisent les traitements cardiovasculaires positifs établis dans des études sur des dizaines de milliers de patients et montrant généralement des réductions modérées du risque relatif, de 15 à $35 \% .^{9}$ Les traitements cardiovasculaires donnent rarement des réductions plus grandes que $35 \%$, parce que ces médicaments ne ciblent habituellement que quelques-uns des nombreux mécanismes pathogéniques sous-jacents à l'affection cardiovasculaire, comme le font les bêta-bloquants périopératoires. ${ }^{6}$

Dans le passé, lorsque les études initiales avaient affiché des avantages remarquablement importants, les études subséquentes démontraient généralement des effets thérapeutiques beaucoup plus modestes - ou même nuls. ${ }^{10,11}$ Ainsi, une méta-analyse d'ERC sur le magnésium dans l'infarctus myocardique a démontré une réduction statistiquement significative de $(P<$ $0,001)>50 \%$ des décès parmi environ 1500 patients randomisés et un nombre modéré de décès (128 décès, ce qui est six fois le nombre total de décès dans les ERC des bêta-bloquants périopératoires). ${ }^{10}$ Cependant, l'ERC subséquente, de 60000 patients environ, n'a montré aucun avantage ; de fait, il y avait une tendance à la hausse de la mortalité avec le magnésium $(P=0,07) .{ }^{11}$

Deuxièmement, peu d'incidents majeurs sont rapportés dans les ERC des bêta-bloquants périopératoires. Si ces médicaments sont efficaces, selon les arguments précités, c'est normal qu'ils démontrent un effet thérapeutique modéré, comme une réduction du risque relatif de $15-35 \%$. Même en posant un taux élevé d'incidents cardiovasculaires de $10 \%$, les essais devraient compter 350 et idéalement 650 événements pour démontrer de façon convaincante une baisse relative du risque de $25 \%$. Or, les données des ERC cités ne comportent que 33 décès cardiovasculaires et des infarctus myocardiques non fatals, ce qui constitue une preuve très limitée. De plus, lorsque la preuve à l'appui découle d'un petit nombre d'études seulement, comportant très peu d'événements, le biais de publication peut sérieusement compromettre la preuve. $^{12}$

Troisièmement, les résultats de la méta-analyse dépendent complètement de ceux d'une seule ERC, par Poldermans et coll., qui a certaines limites. ${ }^{7}$ Réalisée avec seulement 112 patients randomisés, elle 
présentait peu d'événements : 11 décès et neuf infarctus myocardiques non fatals. C'était une ERC ouverte. Or, la recherche démontre que les études ouvertes peuvent introduire des biais plus grands que les effets thérapeutiques qu'on tente de détecter. ${ }^{13}$ L'ERC a été arrêtée parce qu'une analyse provisoire annonçait de grands effets thérapeutiques, par exemple $100 \%$ de réduction du risque relatif pour les infarctus myocardiques non fatals et $80 \%$ de réduction du risque relatif de décès cardiaque. Ce sont des effets invraisemblablement importants qui ne correspondent pas aux résultats des ERC des bêta-bloquants dans les infarctus myocardiques et l'insuffisance cardiaque congestive qui ont randomisé > 50000 patients et démontré des effets modérés, à savoir des réductions du risque relatif de $15-35 \%) .{ }^{14,15}$ Les études qui ont montré que des patients ont subi un infarctus myocardique, malgré un traitement aux bêta-bloquants, réfutent la prétention d'une réduction du risque relatif de $100 \%$ de ces infarctus avec les bêtabloquants. ${ }^{16}$ Finalement, la preuve empirique nous invite à douter des grands effets thérapeutiques inattendus des analyses provisoires. ${ }^{17}$

Certains auteurs ont reconnu les limites de la preuve des ERC sur les bêta-bloquants périopératoires, mais ont soutenu «qu'étant donné leur grande efficacité en prévention secondaire après un infarctus myocardique, des études supplémentaires ne sont pas nécessaires.» ${ }^{18}$ Nos connaissances actuelles de la physiopathologie des événements cardiovasculaires périopératoires sont limitées et il est possible que la physiopathologie des infarctus myocardiques périopératoires diffère de celle des syndromes coronaires aigus non chirurgicaux. L'idée de traiter une affection donnée en se fondant sur le fait qu'un médicament soit bénéfique pour une autre affection de physiopathologie similaire s'est révélée désastreuse dans bon nombre de cas. Par exemple, l'infarctus du myocarde avec élévation du segment $S T$, ou sans élévation du segment ST, de même qu'une angine instable partagent tous une physiopathologie très semblable avec le thrombus coronarien aigu. Le traitement thrombolytique est efficace dans l'infarctus myocardique avec élévation du segment ST, mais non dans les autres conditions. ${ }^{19}$ À partir de ces faits et des complications potentielles (bradycardie et hypotension significatives au plan clinique), et du coût des bêta-bloquants périopératoires, il n'est pas dans le meilleur intérêt du patient de supposer que les données sur la prévention secondaire de l'infarctus myocardique avec des bêta-bloquants constituent une indication de la prise en charge des patients de chirurgie non cardiaque.

Le Dr Rapchuk et ses collègues ont évalué l'utilisa- tion des bêta-bloquants dans «des cas appropriés aux bêta-bloquants périopératoires.» ${ }^{1}$ Ils ont déclaré "avoir utilisé les directives de l'American College of Physicians (ACP) pour choisir leurs patients.» 20 Pourtant, l'ACP n'a pas de directive recommandant le traitement bêta-bloquant périopératoire. Les deux auteurs des directives ont plutôt inséré leurs propres recommandations pour un traitement bêta-bloquant périopératoire après la révision et l'approbation des directives par l'ACP. Les auteurs ont fondé leur recommandation sur les résultats de l'ERC de Mangano et coll., laquelle présente un résultat non significatif au plan statistique selon l'analyse d'intention thérapeutique.

Plus récemment, l'American College of Cardiology/American Heart Association (ACC/AHA) Task Force sur les directives cliniques pour la chirurgie non cardiaque a recommandé le traitement bêta-bloquant pour les patients affectés, ou à risque, de coronaropathie devant subir une intervention non cardiaque. ${ }^{21}$ Le groupe d'étude de l'ACC/AHA a cependant affirmé qu'il «y a encore très peu d'études randomisées d'interventions médicales avant la chirurgie non cardiaque pour prévenir les complications cardiaques périopératoires et elles ne fournissent pas assez de données permettant de formuler des conclusions ou des recommandations fermes.» ${ }^{21}$ D'où la nécessité de distinguer entre elles les recommandations faibles ou fortes. De faibles recommandations sur les bêta-bloquants périopératoires sont similaires aux faibles recommandations antérieures sur l'administration d'hormonothérapie substitutive aux femmes postménopausées. ${ }^{22}$ Les résultats des récentes ERC qui ont démontré que l'hormonothérapie substitutive augmentait les risque de maladie coronarienne nous rappellent les raisons essentielles d'entreprendre de grandes études définitives et d'éviter de s'accommoder des recommandations faibles. ${ }^{23}$

\section{La nécessité d'une grande ERC définitive sur les bêta-bloquants périopératoires}

Étant donné l'évidence prometteuse, mais incertaine, des bêta-bloquants périopératoires, un groupe international de chercheurs s'est réuni et a proposé d'établir définitivement l'effet du traitement bêta-bloquant périopératoire. Le groupe a récemment commencé une ERC sur les bêta-bloquants périopératoires auprès de 10000 patients randomisés. L'étude, appelée PeriOperative ISchemic Evaluation (POISE) trial, est financée par les Instituts de recherche en santé du Canada et le National Health and Medical Research Council subventionné par le Commonwealth Government d'Australie. L'étude POISE randomise 
les patients pour recevoir du métoprolol périopératoire à libération lente, ou un placebo, et l'effet principal noté comprend le décès cardiovasculaire, l'infarctus myocardique non fatal et l'arrêt cardiaque non fatal. Jusqu'à maintenant, l'étude a randomisé 2000 patients de plus de 100 centres dans 11 pays.

\section{Conclusion}

L'argument de la présente ERC, évaluant les effets du traitement bêta-bloquant périopératoire sur les effets cardiovasculaires à court et long terme, présente des limites significatives. Les résultats des ERC doivent être vus comme prometteurs mais tempérés par un scepticisme prudent. Par conséquent, les recommandations sur les bêta-bloquants périopératoires sont faibles parce que la preuve sous-jacente est faible. L'étude POISE fournira une réponse définitive à la détermination de l'efficacité du traitement bêta-bloquant périopératoire.

\section{Remerciements}

Le Dr P. J. Devereaux a apporté une contribution significative au sujet et à la forme du présent article. Il en a écrit la version préliminaire et il a réalisé des révisions critiques du manuscrit. Il a aussi approuvé la version finale de l'article soumis. Les Dr Kate Leslie et Homer Yang ont significativement contribué au contenu et à la forme de l'article et réalisé des révisions critiques du manuscrit. Ils ont aussi approuvé la version finale de l'article soumis.

\section{References}

1 Rapchuk I, Rabuka S, Tonelli M. Perioperative use of beta-blockers remains low: experience of a single Canadian tertiary institution. Can J Anesth 2004; 51: 761-67.

2 Schmidt M, Lindenauer PK, Fitzgerald JL, Benjamin $E M$. Forecasting the impact of a clinical practice guideline for perioperative $B$-blockers to reduce cardiovascular morbidity and mortality. Arch Intern Med 2002; 162: 63-9.

3 Grayburn PA, Hillis LD. Cardiac events in patients undergoing noncardiac surgery: shifting the paradigm from noninvasive risk stratification to therapy. Ann Intern Med 2003; 138: 506-11.

4 Selzman CH, Miller SA, Zimmerman MA, Harken AH. The case for $ß$-adrenergic blockade as prophylaxis against perioperative cardiovascular morbidity and mortality. Arch Surg 2001; 136: 286-90.

5 Stevens RD, Burri H, Tramer MR. Pharmacologic myocardial protection in patients undergoing noncardiac surgery: a quantitative systematic review. Anesth Analg 2003; 97: 623-33
6 Devereaux PJ, Leslie $K$. Best evidence in anesthetic practice: Prevention: $\alpha_{2}$ - and $ß$-adrenergic antagonists reduce perioperative cardiac events. Can J Anesth 2004; 51: 290-2.

7 Poldermans D, Boersma E, Bax JJ, et al. The effect of bisoprolol on perioperative mortality and myocardial infarction in high-risk patients undergoing vascular surgery. Dutch Echocardiographic Cardiac Risk Evaluation Applying Stress Echocardiography Study Group. N Engl J Med 1999; 341: 1789-94.

8 Mangano DT, Layng EL, Wallace A, Tateo I. Effect of atenolol on mortality and cardiovascular morbidity after noncardiac surgery. Multicenter Study of Perioperative Ischemia Research Group. N Engl J Med 1996; 335: 1713-20.

9 Tusuf S, Collins R, Peto R. Why do we need some large, simple randomized trials? Stat Med 1984; 3 : 409-22.

10 Teo KK, Yusuf S, Collins R, Held PH, Peto R. Effects of intravenous magnesium in suspected acute myocardial infarction: overview of randomised trials. BMJ 1991; 303: 1499-503.

11 ISIS-4 (Fourth International Study of Infarct Survival) Collaborative Group. ISIS-4: a randomised factorial trial assessing early oral captopril, oral mononitrate, and intravenous magnesium sulphate in 58050 patients with suspected acute myocardial infarction. Lancet 1995; 345: 669-85.

12 Egger M, Smith GD. Bias in location and selection of studies. BMJ 1998; 316: 61-6.

13 Schulz KF, Chalmers I, Hayes RJ, Altman DG. Empirical evidence of bias. Dimensions of methodological quality associated with estimates of treatment effects in controlled trials. JAMA 1995; 273: 408-12.

14 Yusuf S, Peto R, Lewis J, Collins R, Sleight P. Beta blockade during and after myocardial infarction: an overview of the randomized trials. Prog Cardiovasc Dis 1985; 27: 335-71.

15 Lechat P, Packer M, Chalon S, Cucherat M, Arab T, Boissel JP. Clinical effects of $B$-adrenergic blockade in chronic heart failure. A meta-analysis of double-blind, placebo-controlled, randomized trials. Circulation 1998; 98: 1184-91.

16 Kertai MD, Bax JJ, Klein J, Poldermans D. Is there any reason to withhold $B$ blockers from high-risk patients with coronary artery disease during surgery? (Editorial). Anesthesiology 2004; 100: 4-7.

17 Wheatley K, Clayton D. Be skeptical about unexpected large apparent treatment effects: the case of an MRC AML12 randomization. Control Clin Trials 2003; 24: 66-70.

18 London MJ, Zaugg M, Schaub MC, Spahn DR. Perioperative $B$-adrenergic receptor blockade. 
Physiologic foundations and clinical controversies.

Anesthesiology 2004; 100: 170-5.

19 The TIMI IIIB Investigators. Effects of tissue plasminogen activator and a comparison of early invasive and conservative strategies in unstable angina and non-Qwave myocardial infarction. Results of the TIMI IIIB Trial. Circulation 1994; 89: 1545-56.

20 Palda VA, Detsky AS. Perioperative assessment and management of risk from coronary artery disease. Ann Intern Med 1997; 127: 313-28.

21 Eagle KA, Berger PB, Calkins $H$, et al. ACC/AHA guideline update for perioperative cardiovascular evaluation for noncardiac surgery-executive summary. A report of the American College of Cardiology/American Heart Association Task Force on Practice Guidelines (committee to update the 1996 guidelines on perioperative cardiovascular evaluation for noncardiac surgery). J Am Coll Cardiol 2002; 39: 542-53.

22 Grady D, Rubin SM, Petitti DB, et al. Hormone therapy to prevent disease and prolong life in postmenopausal women. Ann Intern Med 1992; 117: 1016-37.

23 Rossouw JE, Anderson GL, Prentice RL, et al. Risks and benefits of estrogen plus progestin in healthy postmenopausal women. Principal results from the Women's Health Initiative randomized controlled trial. JAMA 2002; 288: 321-33. 